\title{
MANIPULATING THE PITCH SIZE CONSTRAINS THE PLAYERS' POSITIONING DURING UNBALANCED SOCCER SMALL-SIDED GAMES PLAYED BY DIFFERENT AGE GROUPS
}

\author{
Gibson Moreira Praca ${ }^{1}$, André Gustavo Pereira de Andrade ${ }^{1}$, Cristóvão de Oliveira Abreu ${ }^{1}$, \\ Pedro Emilio Drumond Moreira ${ }^{1}$, Filipe Manuel Clemente ${ }^{2,3}$, and Rodrigo Aquino ${ }^{4}$ \\ ${ }^{1}$ Universidade Federal de Minas Gerais, Belo Horizonte, Minas Gerais, Brazil \\ ${ }^{2}$ Escola Superior Desporto e Lazer, Instituto Politécnico de Viana Do Castelo, \\ Viana Do Castelo, Portugal \\ ${ }^{3}$ Instituto de Telecomunicações, Delegação da Covilhã, Lisboa, Portugal \\ ${ }^{4}$ LabSport, Centre of Physical Education and Sport, Federal University of Espírito Santo, \\ Vitória/ES, Brazil
}

Original scientific paper

DOI 10.26582/k.53.2.3

\begin{abstract}
:
We aim to investigate the impact of variable soccer pitch sizes on the position of players during unbalanced small-sided games (SSGs) and compare the responses of players from two different age groups. Forty-eight young players $(n=24$ under-13 and $n=24$ under-14) took part in the study and played 3 vs. $3+1$ SSGs on two different pitch sizes (smaller: 36 × $27 \mathrm{~m} / 139 \mathrm{~m}^{2}$ per player; and larger: 40 x $29 \mathrm{~m} / 166 \mathrm{~m}^{2}$ per player). Players' positions on the pitch (length, width, length-to-width ratio, stretching index, and spatial exploration index) were assessed by the positional data provided by global position system devices and were compared within age groups and between game formats using a two-way analysis of variance. Results showed higher values of spatial exploration index (SEI) in the larger SSGs than in the smaller format $(\mathrm{p}<.001)$. Moreover, a higher length $(p<0.001)$ and length-to-width ratio $(p<.001)$ was observed in the younger group. Finally, the larger format presented higher values of SEI and stretching index, independent of the age group $(\mathrm{p}<.001)$. In the smaller format, U-14 players presented a higher stretching index, while in the larger format U-13 players presented higher values in the same variable. We conclude that enlarging the pitch size constrains players' behaviour during SSGs, and older players are more able to deal with this more complex task context than younger ones.
\end{abstract}

Key words: football, performance, positional analysis, task constraints, small-sided games

\section{Introduction}

Nonlinear pedagogy was proposed as a learning approach based on the manipulation of key tasks and environmental and individual constraints to facilitate the learning of functional and co-adaptive movement patterns through a game-based and player-centred approach (Chow, et al., 2007; Machado, Barreira, et al., 2019; Pizarro, Práxedes, Travassos, \& Moreno, 2020). This teaching model is based on the players' exploration of possible solutions during representative tasks, mainly using small-sided games (SSGs) (Davids, Araújo, Correia \& Vilar, 2013; Ibáñez, Pérez-Goye, García-Rubio \& Courel-Ibáñez, 2020). Consequently, recent studies aimed to investigate how manipulating task constraints such as the pitch size (Moreira, et al., 2020; Silva, Aguiar, et al., 2014), the number of players (Folgado, Lemmink, Frencken \& Sampaio,
2014), and specific rules (Praça, et al., 2021; Sousa, Bredt, Greco, Clemente, \& Praça, 2019) shapes the behaviour of teams and players during SSGs. These data provide significant pieces of information for coaches to adequately plan tasks suitable for different aims and age groups. At this point, the positional analysis, which refers to the analysis of players' occupation and space management on the pitch (Silva, Nguyen, Correia, Clemente, \& Martins, 2019), provides resourceful pieces of information on both individual and collective scales. GPS devices are nowadays available and are used in both training and competition in soccer to gather positional data (Clemente, Afonso, et al., 2020; Praça, et al., 2021) as they provide reliable data on players' displacements (Tessaro \& Williams, 2018). However, despite its usability for analyzing players' tactical positioning and moving, the majority of the liter- 
ature has so far focused on the physical variables (e.g. distances covered and accelerations) provided by such devices. On the other hand, by analysing the impact of changing task constraints on players' position on the pitch and providing coaches with these pieces of information, there is an expectation that the quality will be improved of the players' tactical development through training (Praça, et al., 2021), which is hugely important in youth soccer.

Adopting small-sided games during the tactical and technical training process in youth soccer has been widely recognised in literature (Clemente, et al., 2021) as representative practical contexts that allow the emergence of faster players' responses and the reduction of the reaction time in the game environment (Pizarro, Praxedes, Travassos, Villar \& Moreno, 2019). An intervention study using SSGs showed that players' tactical development was facilitated when numerically unbalanced SSGs were adopted in comparison to numerically equal formats (Praxedes, Moreno, Gil-Arias, Claver, \& Del Villar, 2018). For this reason, many recent studies addressed how manipulating the task constraints, such as the pitch size and the number of players, allow coaches to emphasize different technicaltactical contents during the training process with SSGs (Clemente, Castillo, et al., 2020). For example, a previous study indicated that smaller pitches facilitate the emergence of passing interactions between the players (Moreira, et al., 2020) while another one showed that larger pitches reduced the movement variability (Silva, Aguiar, et al., 2014). Together, these data support the assumption that the manipulation of practice task constraints such as the pitch size modification might be investigated to allow an evidence-based prescription during the training process in soccer.

Previous studies on soccer SSGs adopted numerically unbalanced (e.g. 3 vs. $3+1$ or 5 vs. 3 ) formats to provide players with a more facilitated scenario to keep possession of the ball. Other studies analyzed the impact of changing the relative area per player on players' responses, mainly from a physical point of view (Castellano, Puente, Echeazarra \& Casamichana, 2015; Moreira, et al., 2020). However, it must be considered that every time an additional player is included in a SSG (inside the pitch), the relative area per player is reduced if the pitch size is kept uniform. For this reason, results on the influence of changing the pitch size on players' responses obtained in the numerically balanced formats will not apply to the unbalanced ones. Consequently, investigating how changing the pitch size influences players' individual and collective positional responses in unbalanced SSGs will provide innovative information for coaches and researchers on this topic.

Specifically, numerically unbalanced SSGs favour the ball circulation and reduce physical demands in comparison to the balanced formats
(Castellano, Silva, Usabiaga, \& Barreira, 2016; Moreira, et al., 2020; Praça, De Custódio, \& Greco, 2015).. Moreover, a previous study showed that only players who practised unbalanced formats of SSGs developed tactical skills after an intervention programme (Praxedes, Moreno, Gil-Arias, Claver, \& Del Villar, 2018). These results reinforce the importance of adopting an unbalanced format, mainly in youth soccer, since it provides a facilitated pedagogical scenario for developing players' tactical skills. Previous studies also showed that the area per player constrains players' tactical actions (Folgado, et al., 2014; Silva, Duarte, et al., 2014). At this point, larger pitches increased physical responses and the interpersonal distances during SSGs (Castillo, Raya-González, Manuel Clemente \& Yanci, J. 2020; Frencken, Van der Plaats, Visscher \& Lemmink, K., 2013), indicating that this task constraint indeed impacts players' decision-making during the training. However, to our knowledge, there are no available data on the effect of changing the pitch size on players' tactical positioning in numerically unbalanced goal-scoring SSGs.

Interestingly, when playing the same SSG, older players presented higher area per team values and a higher stretching index (Clemente, Castillo, \& Los Arcos, 2020) and presented higher distances between teams' centroids (Folgado, et al., 2014). Therefore, players' positional dynamics are affected by expertise, so players from different age groups in youth soccer could react differently to the same task constraints. For this reason, the previously reported results regarding the influence of area per player on positional responses in SSG are not generalizable to all age groups and further investigation is required.

As demonstrated, data available in the literature about the influence of pitch size on players' positioning in numerically unbalanced SSGs are scarce. A recent study analysed the impact of varied pitch size on players' behaviour during unbalanced ball possession games (Nunes, Gonçalves, Coutinho, Nakamura, \& Travassos, B., 2020), however, it remains unclear whether these results apply to scoring-goal games. Moreover, the same tasks might present contradictory results when applied to different age groups, and hence generalisations must be avoided. For these reasons, the novelty of the current study assembles on the investigation of the impact of changing the pitch size on positional behaviour during unbalanced SSGs in soccer, which, to the best of our knowledge, has not yet been addressed in literature. Based on this rationale, we aim to compare players' positional responses to SSGs with different pitch sizes, and to compare such responses between players from different age groups in youth soccer (U-13 and U-14). We expect that larger pitch sizes will present higher values of length, width, and spatial exploration than smaller ones, while older players will position themselves 
further away from the game centre, thus increasing the stretching index of the group.

\section{Methods}

\section{Experimental approach to the problem}

This study followed an observational analytical cross-sectional study design. Young players from two different age groups, under-13 and under-14, were recruited. They played SSGs under the same rules (similar to the formal game) on two different pitch sizes: $36 \times 27 \mathrm{~m}$ and $40 \times 29 \mathrm{~m}$. The confrontations were standardised, and all the participants played the same number of SSGs in each format. The SSGs were played always before the main session to reduce the influence of the fatigue on the observed responses. Each SSG lasted four minutes and each session comprised four bouts, with four minutes of passive recovery in-between. During these formats, the data were collected by GPS devices and later analysed to generate positional variables.

\section{Participants}

Forty-eight young soccer players, belonging to two different age groups (U-13: twenty-four players, $13.1 \pm 0.6$ years of age, $6.4 \pm 1.8$ years of soccer experience; U-14: twenty-four players, $14.3 \pm 0.7$ years of age, $9.1 \pm 0.9$ years of soccer practice), took part in the study. The sample size was estimated on the basis of the data provided by a pilot study. In that study, we used the variable with the highest coefficient of variation and adopted an $\alpha$ of 0.05 and a $\beta$ of 0.80 . The results indicated that there was a need for a minimum of sixteen players in each category - lower than the current sample. All players participated regularly in national-level competitions and were selected from a high-standard youth academy. The participants had a training regimen composed of five weekly training sessions plus an official match. All players compete regularly (regional and national levels). The eligibility criteria and methods of selection were as follows: playing regularly in the team, having no injuries or not being in a transition to the field after an injury, and consenting to participate in the data collection. This study was approved by the local ethics committee and followed all procedures of the Declaration of Helsinki.

\section{Procedures}

The procedures comprised three different phases: team composition, familiarisation, and SSGs. All details are presented below.

i) Team composition: Literature shows that the team composition criteria impact players' tactical performance during SSGs (Praça, Morales, et al., 2017). Therefore, we decided to balance the teams based on the tactical skills of the selected players. The players were initially split into groups of eight within the same playing posi- tion and age category (e.g. eight U-14 defenders). Within each position, multiple games were arranged to allow all players to engage in at least one bout of the recommended 3 vs. 3 SSG. Even if a player engaged in more than one bout, only the first one was assessed, and the rest interval was greater than five minutes to reduce the influence of fatigue on the reported performance. These groups played the field test of the System of Tactical Assessment in Soccer (FUTSAT) (Teoldo, Garganta, Greco, Mesquita, \& Maia, 2011), which was used to rank the players from first to eighth within each position. This rank was based on the percentage of successful tactical actions, as previously conducted in the literature (Sousa, et al., 2019). Thereafter, the two best players in each position were selected to form teams A and B, while the two lowest ranked players in each position formed teams $\mathrm{C}$ and D. Midfielders not selected for the teams were selected to play as floaters during the data collection. Finally, the remaining players were kept within the group in case any of the selected players had a problem attending because of, for example, injuries and for the purpose of data collection. The teams played only against opponents at a similar level to reduce the influence of the level of opposition on the observed responses. All procedures related to the team composition are described in Figure 1.

ii) Familiarisation: Players were introduced to each format in one session, and teams were randomly defined as there was no assessment being made. During this session, they could ask questions and experiment with all the rules of each format. After all doubts had been cleared, players were considered to have become familiar with the formats.

iii) SSGs: Every data collection session started with a ten-minute standardised warm-up composed of displacements with and without the ball. The SSG started immediately after the warm-up. All regular rules, including the offside rule, were adopted. Extra balls were placed around the field to allow a quick restart of the game when the ball went out of bounds. We used 7 -a-side soccer goalposts $(6 \times 2 \mathrm{~m})$ during the SSG. Verbal encouragement was allowed by the researcher, but no technical or tactical instructions could be provided. The experimental design comprised a 3 vs. $3+1$ SSG in two different pitch sizes: $36 \times 27 \mathrm{~m}\left(\sim 139 \mathrm{~m}^{2}\right)$ and 40 x $29 \mathrm{~m}$ ( $\sim 166 \mathrm{~m}^{2}, \sim 19 \%$ larger). The $36 \times 27$ $\mathrm{m}$ pitch size was extensively adopted in literature (Machado, Padilha, et al., 2019; Sousa, et al., 2019; Teoldo, et al., 2011) and was reported to represent the same proportions of the official pitch to the 3 vs. 3 format. The SSGs played are represented in Figure 2. On the other hand, the 


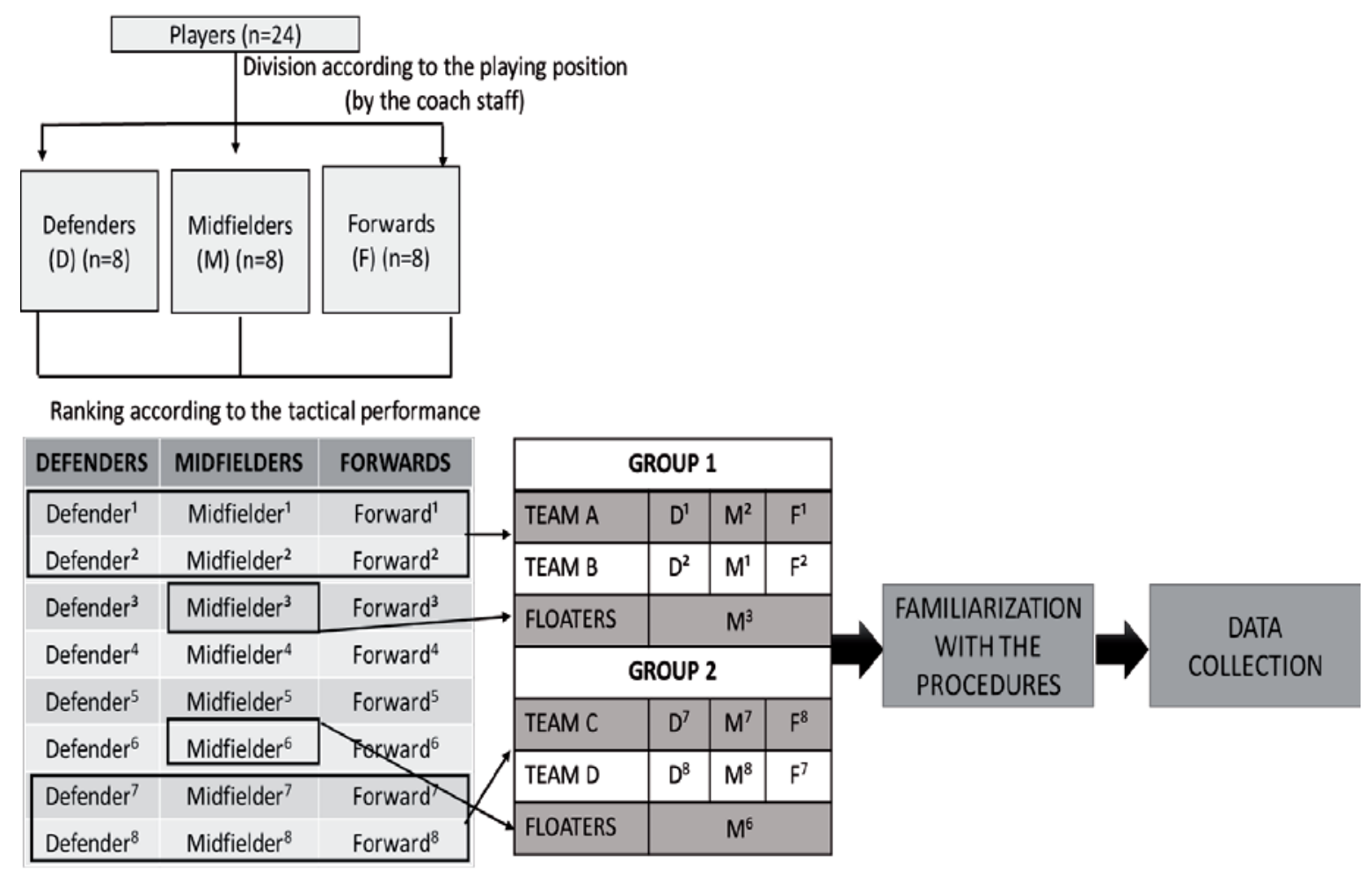

Figure 1. Representation of the procedures of collecting data on each age group.

A

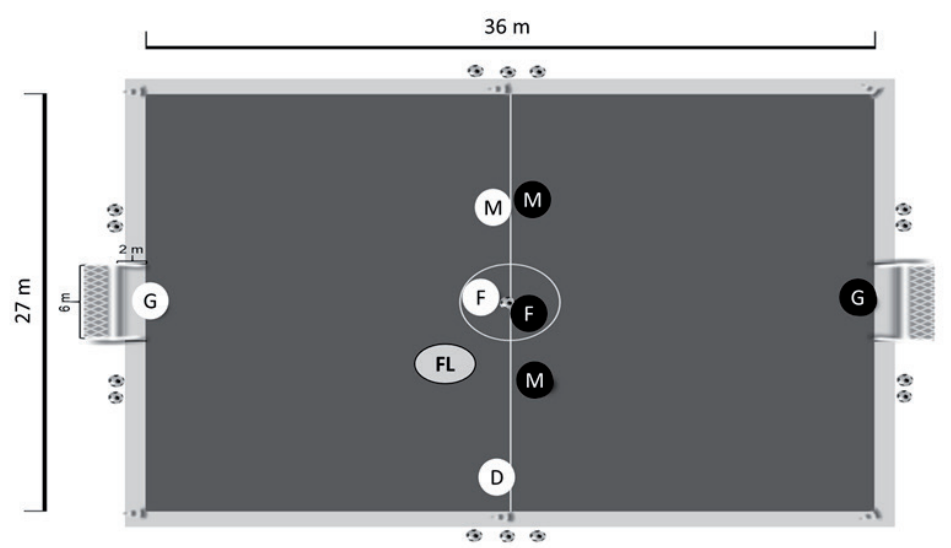

B

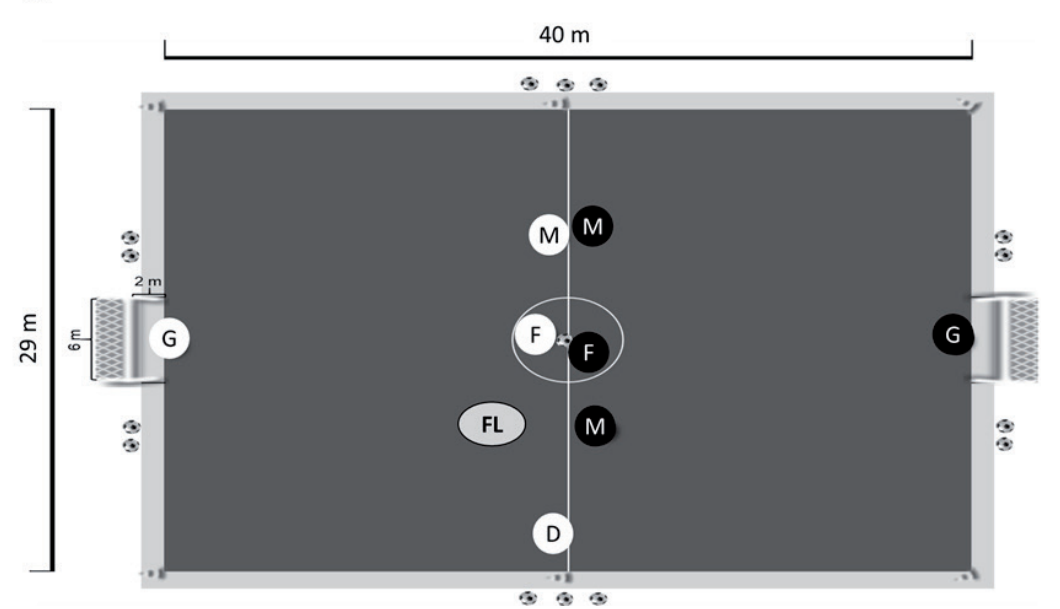

Note. A: 36x27 SSG; B: 40x29 SSG; D: defender; M: midfielder; F: forward; G: goalkeeper; FL: floater.

Figure 2. Representation of the small-sided games. 
relative area per player usually adopted in this pitch is $162 \mathrm{~m}^{2}$, which has been reproduced in the 40 x $29 \mathrm{~m}$ pitch size $\left(\sim 166 \mathrm{~m}^{2}\right.$ considering the seven players). Therefore, in the current study, we tested both absolute and relative areas largely adopted in literature.

In all the SSGs, the additional player functioned as a floater, changing teams whenever the ball possession was recovered by the opposition. This additional player was not allowed to score goals, although he could move freely on the field and perform all other characteristic actions of the game.

\section{Instruments and outcomes}

\section{System of Tactical Assessment in Soccer}

The field test of the FUT-SAT was used for the composition of the teams. The test consists of a 4 minute 3 vs. 3 SSG with goalkeepers in a $36 \times 27$ metre playing field and with $6 \times 2$ metre goalposts (Teoldo, et al., 2011). The tactical behaviour was evaluated based on offensive (penetration, offensive coverage, depth mobility, width and length with and without the ball, and offensive unity) and defensive tactical principles (delay, defensive coverage, balance, concentration, and defensive unity). The percentage of successful tactical principles (ratio between the number of successful tactical principles and the total number of tactical principles performed) was used as a measure (main outcome) of tactical performance (Praça, Morales, et al., 2017).

The SSGs were recorded with a digital camera (JVC, Kenwood, EUA), and two trained observers in FUT-SAT analysed the players' tactical behaviour using the software Soccer Analyser ${ }^{\circledR}$. This software allows the insertion of a virtual grid on the field in the video, setting some parameters for the evaluation of the tactical principles, namely, the offensive and defensive fields, the lateral and central sectors, the game centre, and the ball line. The data were evaluated for inter- and intra-observer agreements. For this, $12.5 \%$ of the SSGs were reassessed 21 days later, and Cohen's Kappa agreement coefficient was calculated. The values of both inter- and intra-observer agreements were higher than 0.8 , which is classified as perfect agreement (Landis \& Koch, 1977).

\section{GPS devices}

The players wore the GPS units, embedded with a triaxial accelerometer (GPSports SPI HPU, GPSports, Canberra, Australia), placed between their shoulder blades, inside a pouch attached to a special vest provided by the manufacturer. Positional data were recorded at $5 \mathrm{~Hz}$ (interpolated to $15 \mathrm{~Hz}$ ). Each player used the same equipment unit during the data collection sessions. The players' positional data were processed using MATLAB R2010a (The MathWorks Inc., Natick, MA, USA). Thereafter, the latitude and longitude data of each player were synchronised and converted into metres using the Universal Transverse Mercator (UTM) coordinate system and a MATLAB routine (Palacios, 2006). The data were smoothed using a second-order $5-\mathrm{Hz}$ Butterworth low-pass filter. After converting the positional data into metres, a rotation matrix was calculated for each SSG with the positions of the field vertices, aligning the length of the playing field with the $\mathrm{x}$-axis and the width along the y-axis. Then, the rotation matrix was applied to players' positional data for alignment with the playing field referential (Folgado, et al., 2014).

The following outcomes were calculated: (i) the width and length, determined by the distance between the furthest players in length and its rightmost and leftmost players in width (ClementeBernardo Sequeiros, Correia, Silva, \& Martins, 2018); (ii) the length per width ratio (LPWratio) (Folgado, et al., 2014); (iii) the stretch index, defined as the average distance from all teammates to the team centroid (Clemente, et al., 2018); and iv) the spatial exploration index (SEI), defined as the average difference between a players' average positions and their actual position at each moment of the game (Clemente, et al., 2018). From a practical perspective, the LPW ratio indicates the preferential displacement axis of a team; values higher than one indicate a more in-length positioning, while values lower than one indicate a more in-width positioning. The stretch index indicates the dispersion of the players from the geometrical centre; higher values indicate higher dispersion. The SEI indicates the exploratory behaviour of the players, and higher SEI values indicate more exploratory behaviour.

\section{Data analysis}

The data were first checked and confirmed for assumptions of normality (Shapiro-Wilk's test) and heterogeneity of the variances (Levene's test). We compared the data using a mixed two-way ANOVA (SSG format $\times$ age group). Pairwise comparisons were performed using Bonferroni's correction. The partial eta squared was calculated for the main effects and classified as small effect: $0.02 \leq \mathrm{h}^{2}{ }_{\mathrm{p}}<$ 0.13 , medium effect: $0.13<\mathrm{h}^{2}<0.26$, or large effect: $\mathrm{h}_{\mathrm{p}}^{2}<0.26$ (Pierce, Block, \& Aguinis, 2004). When interaction effects were found, Cohen's d effect size was calculated for each pairwise comparison and classified as small (0.2) moderate (0.5), or large (0.8) (Cohen, 1988). After this, the inferential analysis was carried out using SPSS (SPSS Version 19.0 for Windows, SPSS Inc., Chicago, IL, USA), and the effect sizes were calculated using GPower software (Version 3.1.9, Franz Faul, Universitat Kiel, Germany). 


\section{Results}

Table 1 shows the values of the analysed variables. The main effect of the protocol $(p<.001$; $\mathrm{h}^{2} \mathrm{p}=0.134$ [medium]) was reported for the SEI, with higher values (10.0\%) being observed in the larger SSGs than in the smaller ones. No age-group differences were reported in this variable $(2.3 \%)$. On the other hand, higher length $\left(23.5 \% ; \mathrm{p}<.001 ; \mathrm{h}^{2} \mathrm{p}=0.258\right.$ [large]) and length-to-width ratio (24.8\%; $<<.001$; $\mathrm{h}^{2} \mathrm{p}=0.160$ [medium]) were reported for U-13, with no between-protocol differences $(0.01 \%$ and $2.9 \%$, respectively). No between-group and betweenformat differences were reported for the width (0.02 and $2.17 \%$, respectively). Finally, an interaction effect was reported for the stretching index $\left(\mathrm{p}<.001 ; \mathrm{h}_{\mathrm{p}}^{2}=0.200\right.$ [medium]). The larger format presented higher values (13.1\%), independent of the age group $\left(\mathrm{p}<.001 ; \mathrm{h}_{\mathrm{p}}^{2}=0.450\right.$ [large]). However, in the smaller format, the U-14 players demonstrated a higher stretching index $(5.76 \%$; $\mathrm{d}=0.879$ [large]), while in the larger format, U-13 players displayed higher values of this variable $(8.28 \% ; \mathrm{d}=0.925$ [large]). literature. In the current study, we showed that a larger pitch size increased players' spatial exploration (high values of SEI and stretching index). Hence, these results indicate that enlarging the pitch increases the displacements and distances between the players during the SSGs. Moreover, these results are congruent with literature since higher interpersonal distances (Frencken, et al., 2013) and higher distances covered (Castillo, et al., 2020) were reported in larger formats. Therefore, similar dynamics emerge in numerically balanced and unbalanced SSG formats. Interestingly, enlarging the pitch size reduces the interactions during the SSGs (Moreira, et al., 2020), which indicates that larger pitches induce a more individual than collective ball-possession strategy. This more individual response tends to be an adaptative behaviour to a more complex task, which seems to be the case when analysing larger pitch sizes. At this point, we advocate that enlarging the pitch size will allow players to be exposed to different tactical problems when playing SSGs, than those experienced in smaller formats, due to the increased

Table 1. Means (standard deviation) of the dependent variables observed in each SSG format and age group

\begin{tabular}{lcccc}
\hline Age group & \multicolumn{2}{c}{ U-13 } & U-14 \\
\hline SSG & Small format & Large format & Small & Large \\
Length * (meters) & $7.88(1.73)$ & $8.06(1.31)$ & $6.55(1.29)$ & $6.35(0.83)$ \\
Width (meters) & $11.97(1.36)$ & $12.34(1.73)$ & $12.11(1.32)$ & $12.26(1.02)$ \\
LPWratio * & $0.67(0.20)$ & $0.66(0.16)$ & $0.55(0.14)$ & $0.52(0.08)$ \\
Stretching Index \#1,2 & $6.18(0.45)$ & $7.48(0.71)$ & $6.54(0.32)$ & $6.91(0.32)$ \\
SEI \# & $8.20(0.97)$ & $8.96(1.08)$ & $7.96(1.05)$ & $8.81(1.05)$ \\
\hline
\end{tabular}

Note. SSG: small-sided game; *: main effect of the category; \#: main effect of the protocol; $1: \mathrm{U}-13>\mathrm{U}-14$ in the larger format; 2: U-14>U13 in the smaller format.

\section{Discussion and conclusion}

Improving the knowledge on the impact of task constraints on players' behaviour during SSGs may enhance the quality of the prescription during the training process in soccer. This is a mandatory issue when considering current learning approaches in team sports, such as nonlinear pedagogy (Chow, et al., 2007). In this sense, we sought to investigate players' responses (in both individual and collective scales) in numerically unbalanced scoring-goal SSGs played by two different age groups when the pitch size was manipulated. In summary, we found that enlarging the pitch size increases the players' spatial exploration and the team's stretching index (irrespective of the age category, although agegroup differences were not similar across the formats). Moreover, younger players presented a higher length and LPW ratio compared with the older players.

The influence of pitch size on players' behaviour during SSGs has widely been broached in the spatial exploration observed. This less predictable scenario will increase the number of available information to be processed by the players and demand more cognitive processing. Considering all these issues, and together with findings from Moreira et al. (2020), we postulate that enlarging the pitch size enhances the complexity of the task in youth soccer and, hence, smaller pitches must be preferably adopted when teaching early age groups. However, we acknowledge that this complexity must not be confounded with the difficulty of the task, as in smaller formats players can experience the need to play faster which can increase the number of errors. This task-complexity enhancement must be considered by coaches together with previously investigated constraints such as game rules (Machado, Ribeiro, et al., 2019), numerical superiority (Praça, Clemente, et al., 2017), presence and number of goals (Ometto, et al., 2018), the adoption of the offside rule (Praça, et al., 2021), and others to an adequate prescription. 
We also investigated how players from different age groups would behave in the SSGs when the pitch size was manipulated. Our results demonstrate that when planning task constraints one must consider players' current level of expertise since age-based differences were observed, which is in line with literature (Almeida, Duarte, Volossovitch, \& Ferreira, 2016; Clemente, et al., 2020). Specifically, older players presented lower values of length than younger players in the current study. At this point, literature has showed that the offside rule constrains players' positioning during SSGs (Praça, et al., 2021) by reducing the in-length displacements. Therefore, the ability to understand the offside rule is likely to explain the current results. Thus, the increased in-length positioning observed might be explained by the reduced ability of younger players to deal with the offside rule during the SSGs. However, we recommend future studies on this topic, based on observational data, to allow the confirmation of such assumption. Conversely, older players tended to position themselves more in the width axis, thus avoiding an offside position. This issue is likely to be more prominent in the numerically unbalanced SSGs since the floater might provide players with safe passing opportunities (Praça, Clemente, et al., 2017). Therefore, positioning predominantly in the width axis may help the players to reinforce the defensive actions and, hence, facilitate keeping the ball possession.

Interestingly, the influence of pitch size on the stretching index of teams was age dependent. In the smaller format, older players presented higher values, which is in line with the current literature (Clemente, et al., 2020). However, younger players presented a higher value in the larger format, contrary to previous findings. At this point, we argue that the current results reinforce the previous rationale that larger pitches increase the complexity of the game, which may discourage adopting larger pitches for younger players. When less experienced players deal with larger formats, they tend to quickly approach the opposing goal by moving along the field depth (Folgado, et al., 2014). Therefore, the increased pitch size harmed players' ability to deal with space occupation, enhancing the stretching index. Conversely, when the pitch size is adequate to their current level of expertise, the expected effect of increasing the stretching index in older groups is observed. Therefore, adjusting the pitch size to players' current skills is manda- tory to optimise tactical development when using SSGs in youth soccer.

This study provided innovative information on how players from different age groups adapt their behaviour during SSGs with different pitch sizes. In addition to its contribution to the area, we acknowledge the need for further research to advance the points not fully addressed by the current study. First, only two age groups were investigated. We, therefore, encourage researchers to enlarge the sample by collecting data from older players (e.g. U-17 and U-20 players). Additionally, we investigated only the 3 vs. $3+1$ format. This SSG format is widely accepted for the early stages of tactical development in soccer because of its ability to represent the tactical context of formal matches (Teoldo, et al., 2011). However, we might consider that the current results do not apply to other formats, mainly when the game requires specific positional characteristics similar to the formal match, such as the 7-a-side match. Therefore, we encourage studies in larger formats to increase the quality of the training prescription.

From a practical perspective, it seems reasonable to propose that coaches consider that changing the pitch size will allow the emphasis on different positional responses during SSGs. Based on the more facilitated scenario observed in the smallest format, we recommend caution when using large SSGs in youth academies as the level of complexity of the task might not be adequate to the players. Also, the coaches might constantly change the pitch to increase the variability of the practice, which seems relevant to develop players' tactical intelligence.

We conclude that larger pitches increase the spatial exploration and stretching index, irrespective of the age category, during numerically unbalanced soccer SSGs. Moreover, older players present a more concentrated game, mainly along the length axis, and increase their ability to explore spaces in reduced pitches. Finally, we advocate that enlarging the pitch size represents a task constraint that might increase the complexity of the game; therefore, it must be cautiously used in youth soccer. Increasing the pitch size for game-based tasks must be a result of long-term investment in the training process. Future studies should search for observational data to confirm that the pitch size indeed represents a way to change the complexity of task at hand. 


\section{References}

Almeida, C.H., Duarte, R., Volossovitch, A., \& Ferreira, A.P. (2016). Scoring mode and age-related effects on youth soccer teams' defensive performance during small-sided games. Journal of Sports Sciences, 34(4), $1355-1362$. https://doi.org/10.1080/02640414.2016.1150602

Castellano, J., Puente, A., Echeazarra, I., \& Casamichana, D. (2015). Influence of the number of players and the relative pitch area per player on heart rate and physical demands in youth soccer. Journal of Strength and Conditioning Research, 29(6), 1683-1691. doi: 10.1519/JSC.0000000000000788

Castellano, J., Silva, P., Usabiaga, O., \& Barreira, D. (2016). The influence of scoring targets and outer-floaters on attacking and defending team dispersion, shape and creation of space during small-sided soccer games. Journal of Human Kinetics, 51(1), 153-163. https://doi.org/10.1515/hukin-2015-0178

Castillo, D., Raya-González, J., Manuel Clemente, F., \& Yanci, J. (2020). The influence of offside rule and pitch sizes on the youth soccer players' small-sided games external loads. Research in Sports Medicine, 28(3), 324-338. https://doi.org/10.1080/15438627.2020.1739687

Chow, J.Y., Davids, K., Button, C., Shuttleworth, R., Renshaw, I., \& Araujo, D. (2007). The role of nonlinear pedagogy in physical education. Review of Educational Research, 77(3), 251-278. https://doi.org/10.3102/003465430305615

Clemente, F.M., Afonso, J., Castillo, D., Arcos, A.L., Silva, A.F., \& Sarmento, H. (2020). The effects of small-sided soccer games on tactical behavior and collective dynamics: A systematic review. Chaos, Solitons and Fractals, 134, 109710. https://doi.org/10.1016/j.chaos.2020.109710

Clemente, F.M., Bernardo Sequeiros, J., Correia, A.F.P.P., Silva, F.G.M., \& Martins, F.M.L. (2018). Computational metrics for soccer analysis: Conecting the dots. Springer. https://doi.org/10.1007/978-3-319-59029-5

Clemente, F.M., Castillo, D., \& Los Arcos, A. (2020). Tactical analysis according to age-level groups during a 4 vs. 4 plus goalkeepers small-sided game. International Journal of Environmental Research and Public Health, 17(5), 1667. https://doi.org/10.3390/ijerph17051667

Clemente, F.M., Ramirez-Campillo, R., Sarmento, H., Praça, G.M., Afonso, J., Silva, A. F., Rosemann, T., \& Knechtle, B. (2021). Effects of small-sided game interventions on the technical execution and tactical behaviors of young and youth team sports players: A systematic review and meta-analysis. Frontiers in Psychology, 12, 667041. https://doi.org/10.3389/fpsyg.2021.667041

Cohen, J. (1988). Statistical power analysis for the behavioral sciences (2nd ed.). Lawrence Erlbaum Associates Publishers.

Davids, K., Araújo, D., Correia, V., \& Vilar, L. (2013). How small-sided and conditioned games enhance acquisition of movement and decision-making skills. Exercise and Sport Sciences Reviews, 41(3), 154-161. doi: 10.1097/ JES.0b013e318292f3ec

Folgado, H., Lemmink, K.A.P.M., Frencken, W., \& Sampaio, J. (2014). Length, width and centroid distance as measures of teams tactical performance in youth football. European Journal of Sport Science, 14(SUPPL. 1), S487-S492. https://doi.org/10.1080/17461391.2012.730060

Frencken, W., Van der Plaats, J., Visscher, C., \& Lemmink, K. (2013). Size matters: Pitch dimensions constrain interactive team behaviour in soccer. Journal of Systems Science and Complexity, 26(1), 85-93. https://doi. org/10.1007/s11424-013-2284-1

Ibáñez, S.J., Pérez-Goye, E., García-Rubio, J., \& Courel-Ibáñez, J. (2020). Effects of task constraints on training workload in elite women's soccer. International Journal of Sports Science and Coaching, 15(1), 99-107. https:// doi.org/10.1177/1747954119891158

Landis, J.R., \& Koch, G.G.C. (1977). The measurement of observer agreement for categorical data. Biometrics, 33(1), 159-174. https://doi.org/10.2307/2529310

Machado, J.C., Barreira, D., Galatti, L., Chow, J.Y., Garganta, J., \& Scaglia, A.J. (2019). Enhancing learning in the context of street football: A case for nonlinear pedagogy. Physical Education and Sport Pedagogy, 24(2), 176-189. https://doi.org/10.1080/17408989.2018.1552674

Machado, G., Padilha, M.B., González Víllora, S., Clemente, F.M., \& Teoldo, I. (2019). The effects of positional role on tactical behaviour in a four-a-side small-sided and conditioned soccer game. Kinesiology, 51(2), 261-270. https://doi.org/10.26582/k.51.2.15

Machado, J.C., Ribeiro, J., Palheta, C.E., Alcântara, C., Barreira, D., Guilherme, J., Garganta, J., \& Scaglia, A.J. (2019). Changing rules and configurations during soccer small-sided and conditioned games. How does it impact teams' tactical behavior? Frontiers in Psychology, 10, 1554. https://doi.org/10.3389/fpsyg.2019.01554

Moreira, P.E.D, Barbosa, G.F., Murta, C.D.C.F., Morales, J.C.P., Bredt, S.D.G.T., Praça, G.M., \& Greco, P.J. (2020). Network analysis and tactical behaviour in soccer small-sided and conditioned games: Influence of absolute and relative playing areas on different age categories. International Journal of Performance Analysis in Sport, 20(1), 64-77. https://doi.org/10.1080/24748668.2019.1705642

Nunes, N.A., Gonçalves, B., Coutinho, D., Nakamura, F.Y., \& Travassos, B. (2020). How playing area dimension and number of players constrain football performance during unbalanced ball possession games. International Journal of Sports Science and Coaching. https://doi.org/10.1177/1747954120966416 
Ometto, L., Vasconcellos, F.V., Cunha, F.A., Teoldo, I., Souza, C.R.B., Dutra, M.B., O’Sullivan, M., \& Davids, K. (2018). How manipulating task constraints in small-sided and conditioned games shapes emergence of individual and collective tactical behaviours in football: A systematic review. International Journal of Sports Science and Coaching, 13(6), 1200-1214. https://doi.org/10.1177/1747954118769183

Palacios, R. (2006). deg2utm. MATLAB Central File Exchange. Downloaded from http://www.mathworks.com/ matlabcentral/fileexchange/10915 on October 20, 2012.

Pierce, C.A., Block, C.A., \& Aguinis, H. (2004). Cautionary note on reporting eta-squared values from multifactor anova designs. Measurement, 64(6), 916-924.

Pizarro, D., Praxedes, A., Travassos, B., del Villar, F., \& Moreno, A. (2019). The effects of a nonlinear pedagogy training program in the technical-tactical behaviour of youth futsal players. International Journal of Sports Science and Coaching, 14(1), 15-23. https://doi.org/10.1177/1747954118812072

Pizarro, D., Práxedes, A., Travassos, B., \& Moreno, A. (2020). Development of defensive actions in small-sided and conditioned games with offensive purposes in futsal. Frontiers in Psychology, 11, 591572. https://doi.org/10.3389/ fpsyg.2020.591572

Praça, G.M., Chagas, M.H., Bredt, S.G.T., Andrade, A.G.P., Custódio, I.J.O., \& Rochael, M. (2021). The influence of the offside rule on players' positional dynamics in soccer small-sided games. Science and Medicine in Football, 5(2), 144-149. https://doi.org/10.1080/24733938.2020.1819559

Praça, G.M., Clemente, F.M., de Andrade, A.G.P., Morales, J.C.P., \& Greco, P.J. (2017). Network analysis in smallsided and conditioned soccer games: The influence of additional players and playing position. Kinesiology, 49(2), 185-193.

Praça, G.M., Custódio, I.J.O., \& Greco, P.J. (2015). Numerical superiority changes the physical demands of soccer players during smallsided games. Revista Brasileira de Cineantropometria e Desempenho Humano, 17(3), 269-279. https://doi.org/10.5007/1980-0037.2015v17n3p269

Praça, G.M., Morales, J.C.P., Moreira, P.E.D., Peixoto, G.H.C., Bredt, S.T., Chagas, M. H., Teoldo, I., \& Greco, P.J. (2017). Tactical behavior in soccer small-sided games- influence of team composition criteria. Revista Brasileira de Cineantropometria e Desempenho Humano, 19(3), 354-363. https://doi.org/10.5007/1980-0037.2017v19n3p354

Praxedes, A., Moreno, A., Gil-Arias, A., Claver, F., \& Del Villar, F. (2018). The effect of small-sided games with different levels of opposition on the tactical behaviour of young footballers with different levels of sport expertise. PLOS ONE, 13(1), e0190157. https://doi.org/10.1371/journal.pone.0190157

Silva, F.G.M., Nguyen, Q.T., Correia, A.F.P.P., Clemente, F.M., \& Martins, F.M.L. (2019). Ultimate Performance Analysis Tool (uPATO). Springer International Publishing. https://doi.org/10.1007/978-3-319-99753-7

Silva, P., Aguiar, P., Duarte, R., Davids, K., Araujo, D., Garganta, J., Araújo, D., \& Garganta, J. (2014). Effects of pitch size and skill level on tactical behaviours of association football players during small-sided and conditioned games. International Journal of Sports Science and Coaching, 9(5), 993-1006. https://doi.org/10.1260/17479541.9.5.993

Silva, P., Duarte, R., Sampaio, J., Aguiar, P., Davids, K., Araujo, D., \& Garganta, J. (2014). Field dimension and skill level constrain team tactical behaviours in small-sided and conditioned games in football. Journal of Sports Sciences, 32(20), 1888-1896. https://doi.org/10.1080/02640414.2014.961950

Sousa, R.B. e, Bredt, S.T., Greco, P.J., Clemente, F.M., \& Praça, G.M. (2019). Influence of limiting the number of ball touches on players' tactical behaviour and network properties during football small-sided games. International Journal of Performance Analysis in Sport, 19(6), 999-1010. https://doi.org/10.1080/24748668.2019.1689751

Teoldo, I., Garganta, J.M., Greco, P.J., Mesquita, I., \& Maia, J. (2011). System of tactical assessment in soccer (FUTSAT): Development and preliminary validation. Motricidade, 7(1), 69-83.

Tessaro, E., \& Williams, J.H. (2018). Validity and reliability of a $15 \mathrm{~Hz}$ GPS device for court-based sports movements. Sports Performance and Science Reports, 29, v1.

Submitted: December 7, 2020

Accepted: May 10, 2021

Published Online First: November 12, 2021

Correspondence to:

Gibson Moreira Praca, Ph.D.

Universidade Federal de Minas Gerais, Brazil

E-mail: gibson_moreira@yahoo.com.br 